\title{
Association of a common genetic variant in prostate stem cell antigen with cancer risk
}

Li Zuo ${ }^{1,2}$, Li Feng Zhang' ${ }^{2}$ Xiao Peng Wu², Zhong Xing Zhou², Jian Gang Zou' ${ }^{2}$ Jun $\mathrm{He}^{3}$, Jian Quan $\mathrm{Hou}^{1}$

\author{
'Department of Urology, The First Affiliated Hospital of Soochow University, Suzhou, China \\ ${ }^{2}$ Department of Urology, Changzhou No. 2 People's Hospital Affiliated to Nanjing \\ Medical University, Changzhou, China \\ ${ }^{3}$ Jiangsu Institute of Hematology, The First Affiliated Hospital of Soochow University, \\ Suzhou, China
}

Submitted: 28 September 2012

Accepted: 6 January 2013

Arch Med Sci 2014; 10, 3: 425-433

DOI: 10.5114 /aoms.2014.43736

Copyright $\odot 2014$ Termedia \& Banach

\section{Abstract}

Introduction: Polymorphisms in the prostate stem cell antigen (PSCA) gene have been hypothesized to increase the genetic susceptibility to cancers. The common sequence variation in PSCA rs2294008 (C>T) has been implicated in cancer risk. However, results of the relevant published studies were somewhat underpowered and controversial in general.

Material and methods: To evaluate the role of PSCA rs2294008 (C>T) genotype in global cancer, we performed a pooled analysis of all the available published studies involving 22,817 cancer patients and 27,753 control subjects.

Results: The results showed evidence that PSCA rs2294008 (C>T) was associated with increased total cancer risk in the overall comparisons. Stratified analysis by cancer type indicated that PSCA rs2294008 T is associated with increased risk of gastric cancer $\left(\mathrm{OR}=1.24,95 \% \mathrm{Cl}=1.09-1.42, p_{\text {he }}\right.$ $\left.<0.001, I^{2}=88.0 \%\right)$ and bladder cancer $(\mathrm{OR}=1.07,95 \% \mathrm{Cl}=1.04-1.11$, $\left.p_{\text {heterogeneity }}=0.108, I^{2}=55.0 \%\right)$ by allelic contrast. Furthermore, in stratified analysis by histological types of gastric cancer, this PSCA variant showed significant associations with diffuse type $(\mathrm{OR}=1.81,95 \% \mathrm{Cl}=1.16-2.81$, $\left.p_{\text {heterogeneity }}<0.001, l^{2}=88.9 \%\right)$ but not intestinal type $(\mathrm{OR}=1.29,95 \% \mathrm{Cl}$ $\left.=0.95-1.74, p_{\text {heterogeneity }}<0.001, l^{2}=85.2 \%\right)$ in a dominant genetic model. Similar results were found in Asian and European descendents and population-based studies.

Conclusions: In all, our meta-analysis suggests that PSCA rs2294008 (C>T) may play allele-specific roles in cancer development. Further prospective studies with larger numbers of participants worldwide should be performed in different kinds of cancer and other descendents in more detail.

Key words: prostate stem cell antigen, polymorphism, cancer risk, metaanalysis.

\section{Introduction}

The prostate stem cell antigen (PSCA) gene encodes a 123-amino-acid glycoprotein with $30 \%$ homology to stem cell antigen 2 (Sca-2), known as a cell surface marker of immature thymic lymphocytes [1]. It was initially identified as the prostate-specific cell-surface antigen over-expressed in prostate cancer, but its expression has subsequently been demonstrated in extraprostatic normal tissues including stomach, esophagus, bladder,
Corresponding author: Jian Quan Hou MD, PhD The First Affiliated Hospital of Soochow University 188 Shizijie St 215006 Suzhou, China Phone: 86051988104931 E-mail: czzzx2000@hotmail.com 
pancreas and kidney [2-5]. In addition to prostate cancer, PSCA is also highly expressed in a proportion of other human solid tumors such as pancreatic, gestational trophoblastic and clear renal cell carcinoma [6-8]. In contrast, reduced PSCA expression was observed in head-and-neck squamous cell, esophagus and stomach tumors $[3,9]$.

It has been shown that PSCA is involved in a variety of tumor growth activities such as cell-proliferation inhibition and/or cell-death induction [10, $11]$, whereas the precise function of this gene remains unknown and controversial. Gu et al. reported that an up-regulated level of PSCA expression was associated with tumor stage, grade and androgen independence of prostate cancer. Conversely, reduced expression of PSCA was found in gastric intestinal metaplasia, a precursor lesion of intestinal-type gastric cancer [12]. In vitro studies indicated that PSCA affects survival of gastric cancer cells, because transfection of PSCA into PSCA-negative cells caused down-regulated cell proliferation [12]. In contrast, down-regulation of PSCA in a human bladder cancer cell line led to a reduction in cell growth, which was associated with activation of several immune signaling pathways [13]. Bahrenberg et al. [3] observed the reduced expression of PSCA in bladder cancer, whereas Amara et al. reported a contradictory result on the association of PSCA expression level and bladder cancer risk [14] It appears that the role of PSCA in tumorigenesis is complex, involving protumorigenic and antitumorigenic functions in various contexts [15].

The PSCA rs2294008, located in the translation starting site which can regulate PSCA promoter activity, is a common genetic variant involved in a variety of studies. Recently, a study group reported findings of a 2-stage genome-wide association study (GWAS), which evaluated the association between rs2294008 single nucleotide polymorphism (SNP) and several histological types of gastric tumor [12]. Then, several independent case-control studies published the association between rs2294008 and other types of carcinoma including prostate cancer [16], bladder cancer [17, 18], upper gastrointestinal cancer [19], breast cancer [20] and colorectal cancer [21]. However, the association between PSCA rs2294008 (C>T) polymorphism and cancer risk is still controversial due to conflicting results from various studies. In this study, a meta-analysis based on 16,306 cancer patients and 17,962 control subjects [12, 16-29] was undertaken to evaluate the effect of PSCA rs2294008 (C>T) polymorphism on cancer risk.

\section{Material and methods}

\section{Literature search and data extraction}

PubMed database searches were performed using the following keywords: 'PSCA' or 'Prostate stem cell antigen', 'polymorphism' and 'cancer' (last search updated on Nov 1, 2012). We also screened references of the retrieved paper and review articles by a hand search. Included studies had to meet the following criteria: (a) information contained the evaluation of PSCA rs2294008 (C>T) polymorphism and cancer risk; (b) case-control studies; (c) involving information about available genotype frequency; (d) only full-text manuscripts were included. The major exclusion criteria were: (a) duplication of previous publications; (b) no available genotype frequency; (c) no control population; (d) study with a clear bias of accrual. Each of the database searches was performed by two of the reviewers to make sure published papers were not missed. Data were refined by the first author's last name, year of publication, study population, ethnicity, genotyping methods, cancer type, sample size in cases and controls, source of control and so on.

\section{Statistical analysis}

Odds ratios (ORs) corresponding to $95 \%$ confidence interval $(\mathrm{Cl})$ were used to evaluate the strength of association based on genotype frequencies in cases and controls. We measured the association between PSCA rs2294008 (C>T) polymorphism and cancer risk by utilizing five genetic contrasts: the allelic contrast ( $T$ allele vs. $C$ allele), the homozygote comparison (TT vs. CC), the heterozygote comparison (TC vs. CC), the dominant genetic model (TT + TC vs. CC) and the recessive genetic model (TT vs. TC + CC). The stratified analysis was carried out by ethnicity, cancer type, source of control (population-based and hospital-based) and histological type. If the cancer type involved only one individual study, it was classified into the "other cancers" subgroup. Hardy-Weinberg equilibrium (HWE) of controls was tested with the Pearson $\chi^{2}$ test for goodness of fit. We used the random effects model and fixed effects model to calculate the pooled OR. The heterogeneity assumption was calculated using a $\chi^{2}$-based $Q$ test among the studies. A $p$-value more than 0.05 for the $Q$ test indicated lack of heterogeneity [30]; thus the fixed effects model (Mantel-Haenszel method [31]) was used. Otherwise, the random effects model (DerSimonian and Laird method [30]) was used. The Z-test was used to examine the statistical significance of the summary $\mathrm{OR}$ and $p<0.05$ was considered significant. Furthermore, $I^{2}$ values were also calculated to better determine the extent of heterogeneity between studies. As a guide, $l^{2}$ values of $<25 \%$ could be considered 'low', values of $\sim 50 \%$ could be considered 'moderate' and values of $>75 \%$ could be considered 'high' [32]. We assessed publication bias using Egger's test and $p<0.05$ was considered 
statistically significant [33]. All statistical analysis was performed using STATA software $v 10.0$ (Stata Corporation, College Station, TX, USA) and all the $p$ values were two sided.

\section{Results}

\section{Study inclusion}

A total of 18 studies (from 15 articles) [12, 1629], which could meet all of the criteria, were included in this study. Genotype distribution of the control population was in line with HWE in each of the studies except for one study conducted in the Polish population [19]. Characteristics of the included studies are summarized in Table I. Among 18 eligible case-control studies, there were 22,817 cases and 27,753 control subjects concerning the PSCA rs2294008 (C>T) polymorphism. There were 11 studies of gastric cancer, three studies of bladder cancer and four studies of other cancers (including prostate cancer, esophageal cancer, breast cancer and colorectal cancer); in the subgroup of ethnicity, 11 were performed in an Asian population, and seven were in European descendents. Population-based controls were carried out in 11 studies. Polymerase chain reaction-restriction fragment length polymorphism (RFLP), the classical genotyping method, was performed in five comparisons. In addition, the GWAS was used in four comparisons.

\section{Quantitative synthesis}

In the overall analysis, a significant association was observed between the PSCA rs2294008 (C>T) and cancer risk in different genetic models: in the contrast of T allele vs. C allele (random-effects OR $=1.17,95 \% \mathrm{Cl}=1.08-1.27, p_{\text {heterogeneity }}<0.001$, $\left.p<0.001, l^{2}=85.0\right)$, the homozygote comparison (random-effects $\mathrm{OR}=1.35,95 \% \mathrm{Cl}=1.13-1.60$, $\left.p_{\text {heterogeneity }}<0.001, p=0.001, l^{2}=86.5\right)$, the heterozygote comparison (random-effects OR = $1.24,95 \% \mathrm{Cl}=1.10-1.40, p_{\text {heterogeneity }}<0.001$, $\left.p=0.001, l^{2}=83.5\right)$, the dominant genetic model (random-effects $\mathrm{OR}=1.28,95 \% \mathrm{Cl}=1.12-1.46$, $\left.p_{\text {heterogeneity }}<0.001, p<0.001, I^{2}=87.3\right)$, the recessive genetic model (random-effects $O R=1.18$, $95 \% \mathrm{Cl}=1.07-1.31, p_{\text {heterogeneity }}<0.001, p=0.001$, $R^{2}=70.1$ ) (Table II). In the stratified analyses by cancer type, an increased risk was found between PSCA rs2294008 (C>T) polymorphism and gastric cancer risk in the allelic contrast (random-effects $\mathrm{OR}=1.24,95 \% \mathrm{Cl}=1.09-1.42, p_{\text {heterogeneity }}<0.001$, $p=0.001, l^{2}=88.0$ ), as well as the homozygote comparison (random-effects OR $=1.50,95 \% \mathrm{Cl}$ $=1.09-2.07, p_{\text {heterogeneity }}<0.001, p=0.012, p^{2}=$ 89.8), the heterozygote comparison (random-effects $\mathrm{OR}=1.40,95 \% \mathrm{Cl}=1.15-1.70, p_{\text {heterogeneity }}$ $\left.<0.001, p=0.001, p^{2}=85.4\right)$ and the dominant genetic model (random-effects OR $=1.45,95 \% \mathrm{Cl}$ $=1.16-1.81, p_{\text {heterogeneity }}<0.001, p=0.001, P^{2}=$ 89.8) (Figure 1). However, in stratified analysis by histological types of gastric cancer, the PSCA variant showed significant associations with diffuse type (dominant genetic model: $O R=1.81$, $95 \% \mathrm{Cl}=1.16-2.81, p_{\text {heterogeneity }}<0.001, p=0.009$, $R^{2}=88.9$; recessive genetic model: $\mathrm{OR}=1.49$, $95 \% \mathrm{Cl}=1.18-1.88, p_{\text {heterogeneity }}=0.014, p=0.001$, $P^{2}=60.1$ ) but not intestinal type (dominant genetic model: $\mathrm{OR}=1.29,95 \% \mathrm{Cl}=0.95-1.74, p$ neity $<0.001, p=0.097, I^{2}=85.2$; recessive genetic model: $\mathrm{OR}=1.11,95 \% \mathrm{Cl}=0.85-1.43, p$ $=0.001, p=0.443, I^{2}=72.7$ ) (Figure 2, Table II). There was also an association between increased risk of bladder cancer and PSCA rs2294008 (C>T) polymorphism in each of the genetic models: in allelic contrast (fixed-effects OR $=1.07,95 \% \mathrm{Cl}=$ $1.04-1.11, p_{\text {heterogeneity }}=0.108, p=0.001, l^{2}=55.0$ ), the homozygote comparison (fixed-effects $\mathrm{OR}=$ $1.15,95 \% \mathrm{Cl}=1.09-1.22, p_{\text {heterogeneity }}=0.190$, $\left.p<0.001, R^{2}=39.8\right)$, the heterozygote comparison (fixed-effects $\mathrm{OR}=1.07,95 \% \mathrm{Cl}=1.05-1.10$, $\left.p_{\text {heterogeneity }}=0.241, p<0.001, l^{2}=29.7\right)$, the dominant genetic model (fixed-effects $O R=1.06$, $95 \% \mathrm{Cl}=1.04-1.09, p_{\text {heterogeneity }}=0.124, p<$ $\left.0.001, l^{2}=52.2\right)$, and the recessive genetic model (fixed-effects $\mathrm{OR}=1.10,95 \% \mathrm{Cl}=1.04-1.16$, $\left.p_{\text {heterogeneity }}=0.293, p=0.001, p^{2}=18.6\right)$. Similar results were also found in Asian descendents and population-based studies, with no significant association observed in the hospital-based studies (Table II). Furthermore, a positive association between this variant and cancer risk in the European population was observed in allelic contrast (random-effects OR $=1.14,95 \% \mathrm{Cl}=1.03-1.26$, $\left.p_{\text {heterogeneity }}<0.001, p=0.009, P^{2}=79.7\right)$, homozygote comparison (random-effects $\mathrm{OR}=1.32,95 \% \mathrm{Cl}=$ $\left.1.09-1.60, p_{\text {heterogeneity }}<0.001, p=0.005, p^{2}=77.5\right)$ and the recessive genetic model (random-effects $\mathrm{OR}=1.22,95 \% \mathrm{Cl}=1.07-1.38, p_{\text {heterogeneity }}=0.017$, $\left.p=0.002, I^{2}=61.2\right)$; the result did not change when excluding the study that was not in HWE.

\section{Publication bias}

We used Begg's funnel plot and Egger's test to evaluate the publication bias of literature. No evidence of publication bias could be found ( $T$ allele vs. C allele, $t=0.19, p=0.853$; TT vs. CC, $t=0.04$, $p=0.966$; TC vs. CC, $t=-0.01, p=0.992$; TT+TC vs. CC, $t=0.19, p=0.851$; TT vs. TC $+C C, t=0.01$, $p=0.989)$.

\section{Discussion}

The increasing growth of human genetics creates countless opportunities for studying disease association. Meta-analysis provides a quantitative method for combining the results from different 


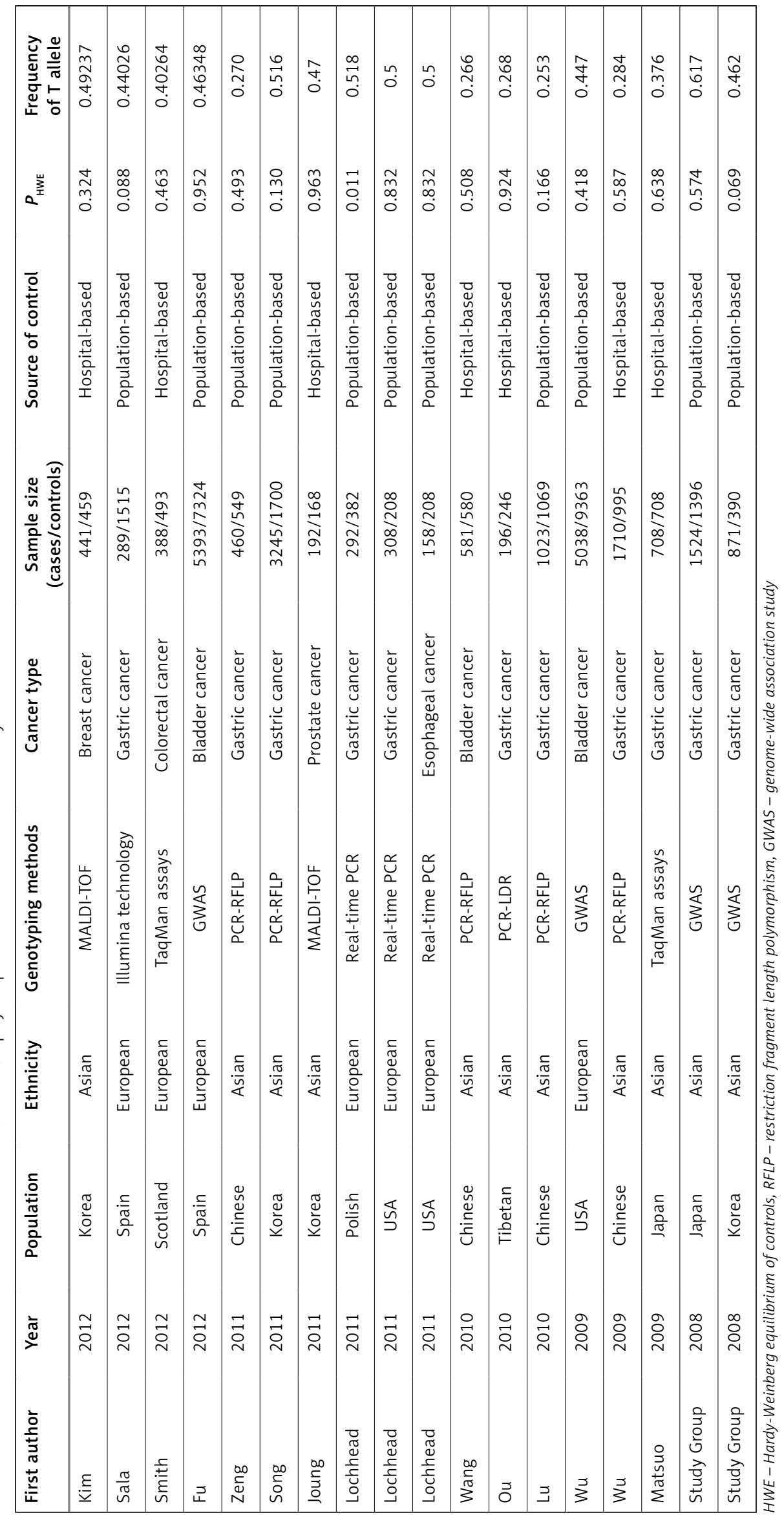




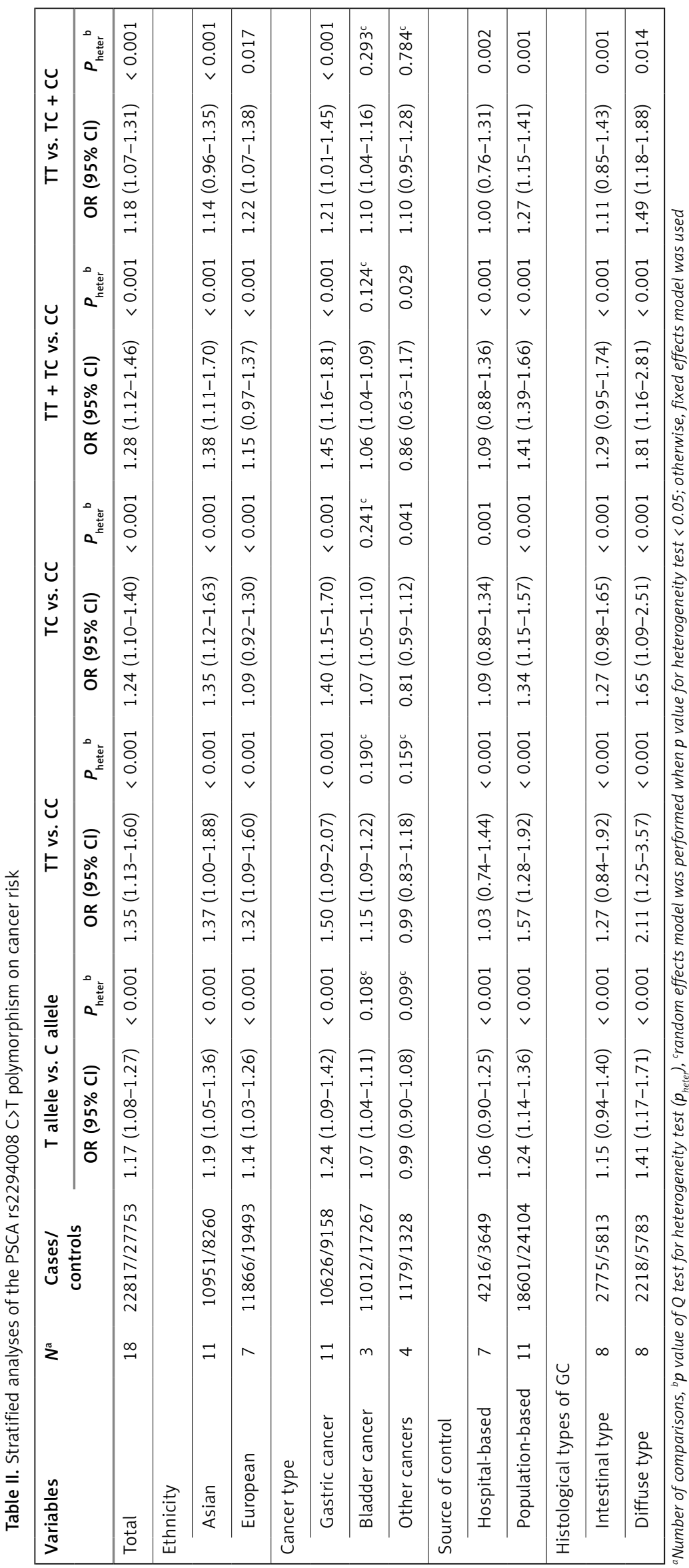




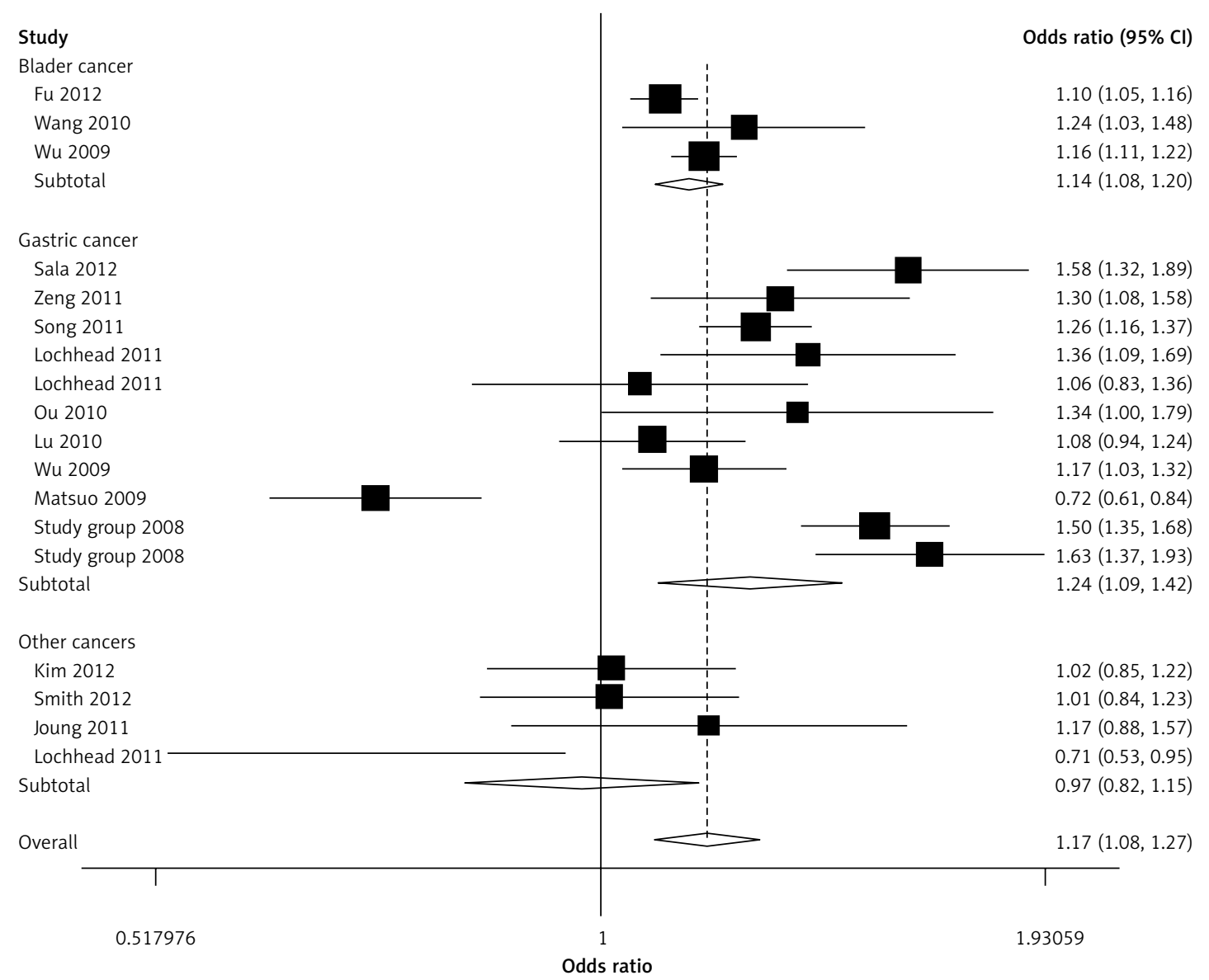

Figure 1. Forest plot of cancer risk associated with the PSCA rs2294008 (C>T) polymorphism (random-effects, T allele vs. $C$ allele) in the stratified analysis by cancer type. The squares and horizontal lines represent the study-specific OR and $95 \% \mathrm{Cl}$. The area of the squares reflects the weight (inverse of the variance). The diamond corresponds to the summary OR and $95 \% \mathrm{Cl}$. See Tables I and II for separate details

studies with the same topic, and for estimating and explaining their diversity [34-36]. Accumulating epidemiological and genetic evidence indicates that genetic variation is a significant component in cancer etiology and the overall goal of this meta-analysis is to combine the results of previous studies in order to achieve summary conclusions about a body of research. To the best of our knowledge, this can be considered as the first pooled analysis to explore the association between PSCA rs2294008 (C>T) polymorphism and overall cancer risk, containing about 22,817 cancer patients and 27,753 control subjects. We found that PSCA rs2294008 (C>T) polymorphism was associated with a significant increased risk of global cancer, especially bladder cancer and gastric cancer. Histopathological research has indicated that gastric cancer is not a single disease and it can be categorized into two major types: intestinal and diffuse. The intestinal-type tumors are found uniformly in geographic areas accompanied with a high incidence of gastric cancer, whereas the diffuse-type are found more predominantly throughout the world [37-40]. Sala et al. performed an EPIC-EURGAST study (based on 148 intestinal gastric cancer cases, 141 diffuse cases and 1515 control subjects) and found that PSCA is associated with both diffuse and intestinal gastric cancer in European [23]. However, our meta-analysis provided evidence that PSCA rs2294008 (C>T) is associated with susceptibility to diffuse type but not intestinal type gastric cancer, which is in line with the former GWAS study [12].

In addition, our results suggested that the effects of PSCA rs2294008 SNPs were stronger in the Asian population than in European descendents. Interestingly, the risk allele (T) frequency was somewhat more common in the Japanese and Korean populations than in the Chinese population. Furthermore, in the stratified analysis by source of control, a significant association between PSCA rs2294008 (C>T) polymorphism and increased cancer risk was observed in population-based studies in each genetic model. Some factors may influence these results. First, it is widely acknowledged that carcinogenesis is a multifactorial procedure induced by complex factors including genetic and environmental inter- 


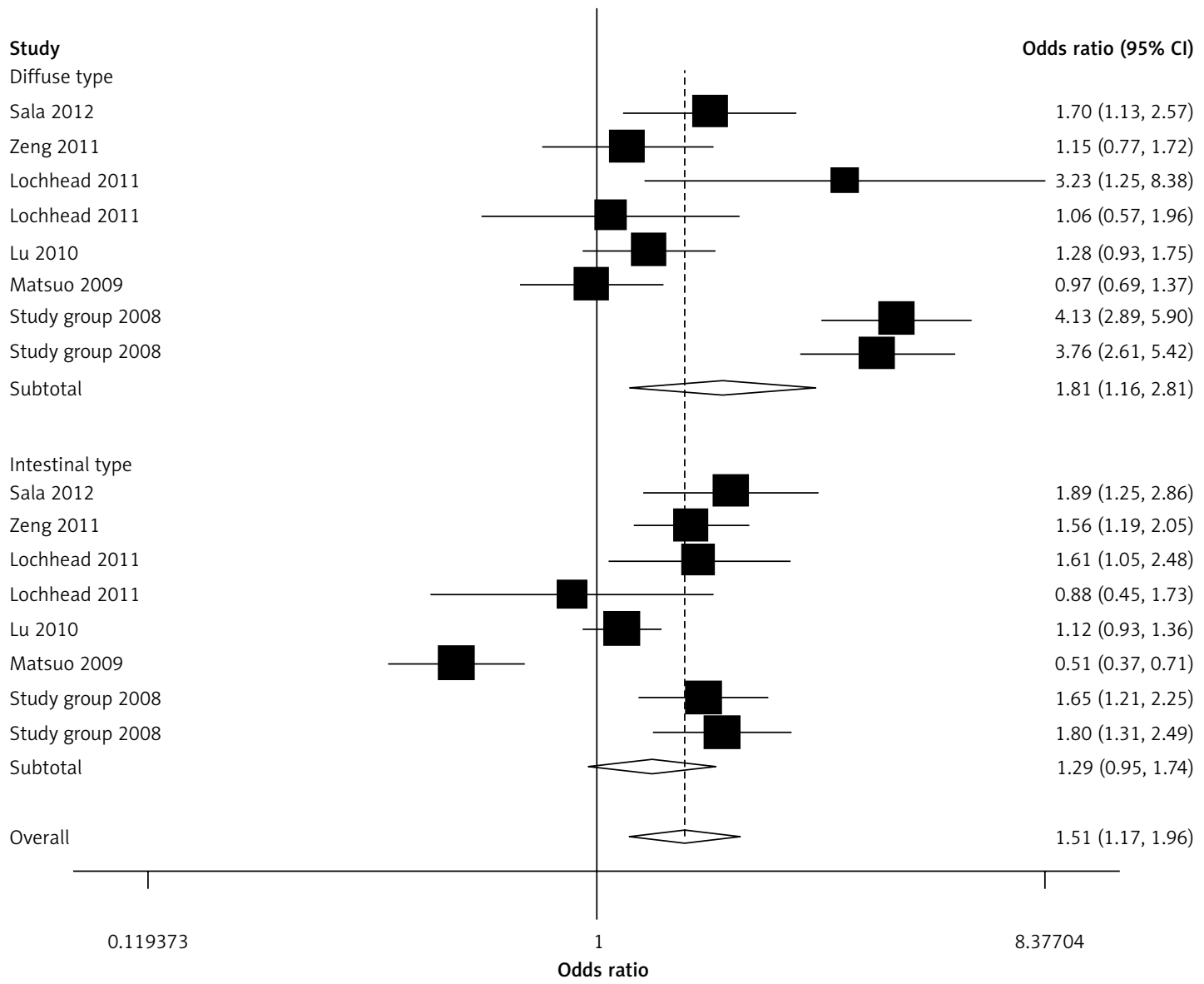

Figure 2. Forest plot of cancer risk associated with the PSCA rs2294008 (C>T) polymorphism (random-effects, dominant genetic model) in the stratified analysis by histological types of gastric cancer. The squares and horizontal lines represent the study-specific OR and $95 \% \mathrm{Cl}$. The area of the squares reflects the weight (inverse of the variance). The diamond corresponds to the summary OR and $95 \% \mathrm{Cl}$

actions (such as dietary factors, age, toxins, radiation, and infectious agents), which indicates that a single factor might have little effect on cancer susceptibility [41]. Second, positive results appear to be published more quickly than articles with negative outcomes, which take longer time to be published (time-lag bias) [42]. Third, discrepancies among the distribution in different ethnicities between case and control populations could be a source of confounding when pooling studies. Furthermore, it is possible that the variant leads to carcinoma at this locus, but the combination of environmental factors or multiple genes [43] may predominate in the development or metastasis of cancer, with the association of the polymorphism not being observed.

Some limitations may exist in the present study. First of all, the numbers of eligible studies accumulated in our analysis may not be sufficiently large for a comprehensive analysis, especially for any given cancer (e.g. prostate cancer, esophageal carcinoma and bladder cancer). Second, our pooled analysis was predominantly based on unadjusted estimates. Thus, more precise anal- ysis including individual information (including smoking, age and sex) should be further conducted if possible. Third, effects based on gene-environment and even different polymorphism loci in the same gene may alter gene expression and account for fluctuation in OR values. However, some key advantages in this present study should also be acknowledged. First, a substantial number of cancer patients and control subjects in this article were included from different studies; therefore, statistical power of the analysis could be significantly increased. Second, no evidence of publication bias was found in this article and quality of the case-control studies was uniformly good. Third, studies included in the meta-analysis strictly and satisfactorily met the selection criteria.

In conclusion, this pooled analysis showed evidence that PSCA rs2294008 (C>T) polymorphism was associated with increased cancer risk and this variant may play allele-specific roles in cancer development. Further prospective studies with larger sizes of unbiased-matched homogeneous participants and well-matched controls worldwide are required to evaluate the association 
between PSCA rs2294008 (C>T) polymorphism and cancer risk to establish more comprehensive conclusions.

\section{Acknowledgments}

This work was supported by the Science Development Foundation of Nanjing Medical University (2011NJMU086), the Society Development Foundation of Changzhou (CS2008917), the National Natural Science Foundation of China (81072085), The Key Laboratory Foundation of Suzhou (No: SZS201001).

\section{References}

1. Reiter RE, Gu Z, Watabe T, et al. Prostate stem cell antigen: a cell surface marker overexpressed in prostate cancer. Proc Natl Acad Sci USA 1998; 95: 1735-40.

2. Gu Z, Thomas G, Yamashiro J, et al. Prostate stem cell antigen (PSCA) expression increases with high gleason score, advanced stage and bone metastasis in prostate cancer. Oncogene 2000; 19: 1288-96.

3. Bahrenberg G, Brauers A, Joost HG, Jakse G. Reduced expression of PSCA, a member of the LY- 6 family of cell surface antigens, in bladder, esophagus, and stomach tumors. Biochem Biophys Res Commun 2000; 275: 783-8.

4. Argani P, Rosty C, Reiter RE, et al. Discovery of new markers of cancer through serial analysis of gene expression: prostate stem cell antigen is overexpressed in pancreatic adenocarcinoma. Cancer Res 2001; 61: 4320-4.

5. Elsamman EM, Fukumori T, Tanimoto S, et al. The expression of prostate stem cell antigen in human clear cell renal cell carcinoma: a quantitative reverse transcriptase-polymerase chain reaction analysis. BJU Int 2006; 98: 668-73.

6. Elsamman E, Fukumori T, Kasai T, et al. Prostate stem cell antigen predicts tumour recurrence in superficial transitional cell carcinoma of the urinary bladder. BJU Int 2006; 97: 1202-7.

7. Grubbs EG, Abdel-Wahab Z, Tyler DS, Pruitt SK. Utilizing quantitative polymerase chain reaction to evaluate prostate stem cell antigen as a tumor marker in pancreatic cancer. Ann Surg Oncol 2006; 13: 1645-54.

8. Feng HC, Tsao SW, Ngan HY, et al. Overexpression of prostate stem cell antigen is associated with gestational trophoblastic neoplasia. Histopathology 2008; 52: 167-74.

9. de Nooij-van Dalen AG, van Dongen GA, Smeets SJ, et al. Characterization of the human Ly- 6 antigens, the newly annotated member Ly-6K included, as molecular markers for head-and-neck squamous cell carcinoma. Int J Cancer 2003; 103: 768-74.

10. Saffran DC, Raitano AB, Hubert RS, Witte ON, Reiter RE, Jakobovits A. Anti-PSCA mAbs inhibit tumor growth and metastasis formation and prolong the survival of mice bearing human prostate cancer xenografts. Proc Natl Acad Sci USA 2001; 98: 2658-663.

11. Gu Z, Yamashiro J, Kono E, Reiter RE. Anti-prostate stem cell antigen monoclonal antibody $1 \mathrm{G} 8$ induces cell death in vitro and inhibits tumor growth in vivo via a Fc-independent mechanism. Cancer Res 2005; 65: 9495-500.

12. Study Group of Millennium Genome Project for Cancer. Genetic variation in PSCA is associated with susceptibil- ity to diffuse-type gastric cancer. Nat Genet 2008; 40: 730-40.

13. Marra E, Uva P, Viti V, et al. Growth delay of human bladder cancer cells by prostate stem cell antigen down-regulation is associated with activation of immune signaling pathways. BMC Cancer 2010; 10: 129.

14. Amara N, Palapattu GS, Schrage M, et al. Prostate stem cell antigen is overexpressed in human transitional cell carcinoma. Cancer Res 2001; 61: 4660-5.

15. Saeki N, Gu J, Yoshida T, Wu X. Prostate stem cell antigen: a Jekyll and Hyde molecule? Clin Cancer Res 2010; 16: 3533-8.

16. Joung JY, Lee YS, Park S, et al. Haplotype analysis of prostate stem cell antigen and association with prostate cancer risk. J Urol 2011; 185: 2112-8.

17. $\mathrm{Wu}$ X, Ye Y, Kiemeney LA, et al. Genetic variation in the prostate stem cell antigen gene PSCA confers susceptibility to urinary bladder cancer. Nat Genet 2009; 41: 991-5.

18. Wang S, Tang J, Wang M, Yuan L, Zhang Z. Genetic variation in PSCA and bladder cancer susceptibility in a Chinese population. Carcinogenesis 2010; 31: 621-4.

19. Lochhead P, Frank B, Hold GL, et al. Genetic variation in the prostate stem cell antigen gene and upper gastrointestinal cancer in white individuals. Gastroenterology 2011; 140: 435-41.

20. Kim SY, Yoo JY, Shin A, et al. Prostate stem cell antigen single nucleotide polymorphisms influence risk of estrogen receptor negative breast cancer in Korean females. Asian Pac J Cancer Prev 2012; 13: 41-8.

21. Smith C, Lochhead P, Basavaraju U, et al. Lack of association between the rs2294008 polymorphism in the prostate stem cell antigen gene and colorectal neoplasia: a case-control and immunohistochemical study. BMC Res Notes 2012; 5: 371.

22. Fu YP, Kohaar I, Rothman N, et al. Common genetic variants in the PSCA gene influence gene expression and bladder cancer risk. Proc Natl Acad Sci U S A 2012; 109: 4974-9.

23. Sala N, Munoz X, Travier N, et al. Prostate stem-cell antigen gene is associated with diffuse and intestinal gastric cancer in Caucasians: results from the EPICEURGAST study. Int J Cancer 2012; 130: 2417-27.

24. Wu C, Wang G, Yang M, et al. Two genetic variants in prostate stem cell antigen and gastric cancer susceptibility in a Chinese population. Mol Carcinog 2009; 48: 1131-8.

25. Matsuo K, Tajima K, Suzuki T, et al. Association of prostate stem cell antigen gene polymorphisms with the risk of stomach cancer in Japanese. Int J Cancer 2009; 125: 1961-4.

26. Ou J, Li K, Ren H, Bai H, Zeng D, Zhang C. Association and haplotype analysis of prostate stem cell antigen with gastric cancer in Tibetans. DNA Cell Biol 2010; 29: 319-23.

27. Lu Y, Chen J, Ding Y, et al. Genetic variation of PSCA gene is associated with the risk of both diffuse- and intestinal-type gastric cancer in a Chinese population. Int J Cancer 2010; 127: 2183-9.

28. Zeng Z, Wu X, Chen F, et al Polymorphisms in prostate stem cell antigen gene rs2294008 increase gastric cancer risk in Chinese. Mol Carcinog 2011; 50: 353-8.

29. Song HR, Kim HN, Piao JM, et al. Association of a common genetic variant in prostate stem-cell antigen with gastric cancer susceptibility in a Korean population. Mol Carcinog 2011; 50: 871-5.

30. DerSimonian R, Laird N. Meta-analysis in clinical trials. Control Clin Trials 1986; 7: 177-88. 
31. Mantel N, Haenszel W. Statistical aspects of the analysis of data from retrospective studies of disease. J Natl Cancer Inst 1959; 22: 719-48.

32. Higgins JP, Thompson SG, Deeks JJ, Altman DG. Measuring inconsistency in meta-analyses. BMJ 2003; 327: 557-60.

33. Egger M, Davey Smith G, Schneider M, Minder C. Bias in meta-analysis detected by a simple, graphical test. BMJ 1997; 315: 629-34.

34. Cheng G, Xie L. Alcohol intake and risk of renal cell carcinoma: a meta-analysis of published case-control studies. Arch Med Sci 2011; 7: 648-57.

35. Ehteshami-Afshar S, Nikfar S, Rezaie A, et al. A systematic review and meta-analysis of the effects of inflix imab on the rate of colectomy and post-operative complications in patients with inflammatory bowel disease. Arch Med Sci 2011; 7: 1000-12.

36. Smolinska K, Paluszkiewicz P. Risk of colorectal cancer in relation to frequency and total amount of red meat consumption. Systematic review and meta-analysis. Arch Med Sci 2010; 6: 605-10.

37. Crew KD, Neugut Al. Epidemiology of gastric cancer World J Gastroenterol 2006; 12: 354-62.

38. Lauren P. The two histological main types of gastric carcinoma: diffuse and so-called intestinal-type carcinoma. An attempt at a histo-clinical classification. Acta Pathol Microbiol Scand 1965; 64: 31-49.

39. Hohenberger P, Gretschel S. Gastric cancer. Lancet 2003, 362: 305-15.

40. Li ZX, Kaminishi M. A comparison of gastric cancer between Japan and China. Gastric Cancer 2009; 12: 52-3.

41. Pharoah PD, Dunning AM, Ponder BA, Easton DF. Association studies for finding cancer-susceptibility genetic variants. Nat Rev Cancer 2004; 4: 850-60.

42. Ioannidis JP. Effect of the statistical significance of results on the time to completion and publication of randomized efficacy trials. JAMA 1998; 279: 281-6.

43. Wei $\mathrm{Q}$, Cheng L, Amos Cl, et al. Repair of tobacco carcinogen-induced DNA adducts and lung cancer risk: a molecular epidemiologic study. J Natl Cancer Inst 2000; 92 1764-72. 